Gazi University
Journal of Science
$\mathrm{http} / / /$ dergipark.gov.tr/gujs

\title{
An Application of a Semi Markov Model in Predicting the Fading Occurrences in Wireless Communication Channels
}

\author{
Shaghayegh KORDNOORI ${ }^{1, *}$ (D) Hamidreza MOSTAFAEI $^{2}$ (D) , Mohammadhassan BEHZADI $^{3}$ \\ ${ }_{1,3}$ Department of Statistics, Science and Research Branch, Islamic Azad University, Tehran, Iran. \\ ${ }^{2}$ Department of Statistics, North Tehran Branch, Islamic Azad University, Tehran, Iran. Email: h_mostafaei@iau-tnb.ac.ir
}

\section{Highlights}

- A new mathematical stochastic method for predicting the future signal fading is introduced.

- The elements of the Semi Markov Model are presented.

- The forecasting of the fading occurrences in dimensions of time and amplitude together is performed.

- The occurrences of deep fades are forecasted for the next 1 to 21 unit times.

\section{Article Info}

Received: $17 / 01 / 2019$

Accepted: 16/10/2019

\section{Keywords}

Semi Markov Model

Holding time

Interval transition probability

Fading occurrence

\begin{abstract}
The most frustrating and troublesome issue in wireless communication is fading. Estimating the fading occurrence for the design of greatly reliable communication link is crucial. In this paper, we present a novel mathematical method for predicting the future signal fading on the basis of current and past data. The application of a Semi Markov Model as a generalization of the Markov Model is discussed for predicting the deep fading occurrence probabilities of the received envelope in wireless communications channels. This flexible model is given for assessing the system performance with the envelope correlation. 142 deep fading data whose amplitudes are lower than a mean of the amplitudes which occurred in a typical wireless system with the Jakes filtering are considered. The transition probability matrix and the holding time mass functions are calculated for the next 1 to 21 unit times. One unit time is regarded as the inverse of sampling frequency; moreover, the core matrix and the cumulative probability distribution of the waiting time are obtained. Calculating the interval transition probabilities for Amplitude to Amplitude transition for these deep fades demonstrates the forecasting occurrence probabilities in the future and the possibility of forecasting the fading occurrences in dimensions of time and amplitude.
\end{abstract}

\section{INTRODUCTION}

In wireless systems, it would be helpful if the behavior of fading could be predicted, applying measurements of past and present behavior. Forecasting the fading behavior has auspicious applications in controlling the simple combining methods in cheap receivers in which informing about the occurrences of fades ahead of time could be beneficial. The information about wireless fading channels is essential in how the wireless communication system is designed.

A powerful mathematical model for predicting the fading channel state is a Markov chain which is a stochastic process in which the future evolution of the system given the current state is independent of its history. This process is usually employed, due to its consolidated theory. The Markov models are useful for the systems with common cause degradation and failures such as signal fading in wireless communication systems. This method was used to forecast the statistical features and variations of channel parameters caused by the changing morphology of narrowband propagation channel. In the Markov model, the next state can be identified by a transition probability matrix. However, Semi-Markov processes (SMPs) are a broad class of stochastic processes which overgeneralize the Markov and renewal processes at the 
same time which are applied in many fields such as computer science [1], system reliability [2], finance [3], biology [4], seismic risk analysis [5], insurance [6], wireless networks [7-9], etc.

In this paper, we regard a fading occurrence prediction as a probabilistic outline about the future fades. In section 2, we review the literature and illustrate the comparison between the mentioned approaches and our proposed model. In section 3, we give a brief description of the fading process. Next, we propose the Semi Markov model. In section 5, we employ this stochastic model to deep fades occurred in a sample wireless system and forecast the probabilities of fading occurrences by the interval transition probabilities for amplitude to amplitude transitions of these fades in different unit times. At last, the conclusions are given.

\section{THE LITERATURE REVIEW AND COMPARISON OF THE PROPOSED MODEL WITH OTHER MODELS}

The most popular approaches are Sum-of-Sinusoids $(\mathrm{SoS})$ techniques and linear prediction procedures rested on Autoregressive (AR) modeling. In [10] the SoS method was applied to predict the fading channel state. In their work, given frequency estimation, three sinusoidal LMMSE predictors were given; moreover, a Joint Moving Average and Sinusoidal prediction model and the associated joint Least-Square predictor were proposed. SoS channel models attempt to simulate the channel as a stationary complex Gaussian random process. They considered this stochastic method for long-range channel prediction. In comparison with our proposed model, The Semi-Markov process (SMP) is more general than Gaussian process as in the Gaussian one every finite collection of the random variables has a multivariate normal distribution, but in the SMP the random variables can take different types of distributions; moreover, the SMP has this ability that can predict the future behavior in short and long-range together. In [11] a spectral estimation was employed which followed by Linear Prediction (LP) and interpolation to predict the fading signals; moreover, they used the AR model and the Maximum Entropy Method for the long-range prediction. In comparison with our proposed model, their work is based on the time series analysis which only depends on the time of a prediction but in our proposed model the duration times in different states, as well as the times of prediction are considered; moreover, we predict both the short and long range of future behavior. In [12] logistic regression analysis was applied to develop an empirical formula for forecasting fading probability. Their work only concerned with forecasting whether or not fading will occur while with the SMP in addition to forecast the occurrence of fading, its probabilities in different unit times with various amplitude can be calculated. In [13] the fast fading mobile channel using a moving average filter was analyzed and then the probability and cumulative distribution functions of the data were calculated. In [14] the inverse discrete Fourier transform, the filtering White Gaussian noise and the SoS methods were discussed for analyzing the fading process. They compared these methods using several quantitative measures. In [15] a multiparametric stochastic approach was employed for forecasting fading phenomena by introducing a new probability distribution function. In [16] an ARMA(p,q) model parameterization method was proposed for multipath Rayleigh fading using the Minimum Description Length criterion. In [17] a suitable model structure was introduced for describing the fading of the radio channel which is a mixture of gamma and compound gamma-lognormal distributions. In [18] and [19] a Markov chain was applied for analyzing the prediction of the fade occurrences. The main problem for the probability distribution fitting mentioned methods is that they can only evaluate the short range of future behavior and can forecast the frequency of occurrence of the fading magnitude in a certain interval. In [20] the dynamics of a correlated Rayleigh fading channel were analyzed with a Markov double-chain for the random fading process with the Markov states corresponding to the quantized amplitude levels. In [21] a four-state Semi Markov model was suggested according to the impairments as a line of sight, shadowing, blockage and the state in which the fade caused by a large obstacle for the Land Mobile Satellite propagation channel. They obtained the fading Semi Markov parameters for an urban, suburban, heavily and lightly wooded environments and regarded the power-law and lognormal distribution as a holding time function. In our Semi Markov model, the states are according to the amplitudes of the signal and we examine the fluctuations of the signal itself and not the impairments. In [22] a finite-state phase-type Semi Markov channel (FSPHMC) was proposed for modeling the correlated fading channel. They considered the phasetype $(\mathrm{PH})$ distributed sojourn time in each state; moreover, for facilitating the implementation of their proposed model, the FSPHMC model with Negative binomial sojourn time which is a special case of PHdistribution was also presented. The fading characteristics such as fade duration distribution and level 
crossing number distributions were captured by this proposed model. In our proposed model we do not consider any restrictions on the use of a special distribution as a holding time and regard the generalization of Wang's work.

All in all, our proposed Semi Markov model forecasts the fading behavior in a novel manner which is different from all the previous studies. We extend the paper [22] and do not consider any special probability distributions such as lognormal or $\mathrm{PH}$; moreover, we forecast the fade occurrences by the interval transition probabilities with respect to different times while none of the previous papers did anything like our work. The proposed method provides a flexible and versatile solution to model various fading processes arising from a wireless communication area with high precision and we illustrate this application for the first time in predicting the future behavior of the fading process.

\section{DESCRIPTION OF THE FADING PROCESS}

Wireless networks play an important role in today's life. One of the most complicated and provocative issues in wireless communication is fading. A signal fading is occurring when there is a quick change in received signal strength over a small-time interval or travel distance. It changes the frequency of a signal. The received signal in wireless communication systems involves remarkable power fluctuations which are the result of multipath propagation existence and the Doppler frequency shift. Fading is often modeled as a stochastic process and deep fades occur occasionally. Fading mathematical modeling is a crucial topic over the last decades. Fading envelope forecasting can enhance the performance of adaptive modulation techniques that need timely channel state information for optimal enforcement. It is vital to transmit the signal to the receiver, effectively. This knowledge can be helpful in choosing a suitable transmission policy and allocating resources. It is important to estimate the deep fading occurrences for the design of a greatly reliable communication link.

\section{The SEMI MARKOV MODEL}

\subsection{Benefits of the Semi Markov Model}

The time spent in any state in a Markov model has to follow an exponential distribution, and that may result in unrealistic values of the duration of the states. The generalization of this process is the Semi Markov Models which are general, powerful and simple enough to capture the main features and specify the short and long run behavior; moreover, the simple interpretation and mathematical tractability are the main advantages of these Models. They are non-poissonian with a renewal property and can obtain the statistical characteristics of the sojourn time in the fading process. See [23-25] for studying SMP in details. In the SMPs, the sojourn time between transitions occurs stochastically according to any kind of distribution functions which depends on the current and next visited state. The SMP contains full information about the process. One way of improving the statistics of duration is to eliminate the Markov model self-loops and achieve the duration in each state from a determined distribution function. In Semi Markov model, the amplitude of a signal at any time is dependent on the amplitude of it on the previous unit time and their time interval between them; moreover, the time between fades has an influence on the pattern of their happening. Hence, the fading occurrence can associate with the Semi Markov models. Utilizing this model would be able us to estimate the fading occurrence probabilities and could be beneficial in depicting a dependence in a sequence of events.

\subsection{The Theoretical Study}

A discrete time SMP is a probabilistic model which is beneficial in modeling the complex dynamic systems. It can model situations much more realistic and can reduce the prediction error. The SMP is constructed by a Markov Renewal Process (MRP) that is a particular case of Markov sequence. In this model, the transition probability relies not only on the present state and time but also on the time spent on that state. The sojourn time of the SMP can be depicted by the arbitrary distribution which is the superiority as compared to the Markov Model which only described by the memoryless distributions. Suppose $0=p_{0} \leq p_{1} \leq \cdots \leq p_{n} \leq$ $p_{n+1} \leq \cdots$ be the jump times of jump stochastic process $Z=\left(Z_{t}\right)_{t \in R^{+}}$with a discrete state space $E$ and 
$J_{0}, J_{1}, J_{2}, \ldots$ are the consecutive visited states of $Z$. Letting $N_{0}$ be the set of nonnegative integers, the stochastic process $\left(U_{n}, P_{n}\right)_{n \in N_{0}}$ is the MRP if it satisfies

$$
P\left(J_{n+1}=j, P_{n+1}-P_{n} \leq t \mid J_{0}, \ldots, J_{n} ; P_{1}, \ldots P_{n}\right)=P\left(J_{n+1}=j, P_{n+1}-P_{n} \leq t \mid J_{n}\right) .
$$

The chain $Z$ is said to be a Semi Markov chain associated with the (MRP) $\left(J_{n}, P_{n}\right)_{n \in N_{0}}$ if

$Z_{t}=J_{N(t)}, t \in N$

where

$$
\begin{aligned}
& N(t)=\max \left\{n \in N \mid P_{n} \leq k\right\} \\
& X_{n}=p_{n}-p_{n-1}
\end{aligned}
$$

is the discrete time counting process of the number jumps in $[1, \mathrm{k}] \subset N$. Thus, $Z_{t}$ gives the system state at time $k$. Figure 1 indicates the SMP path.

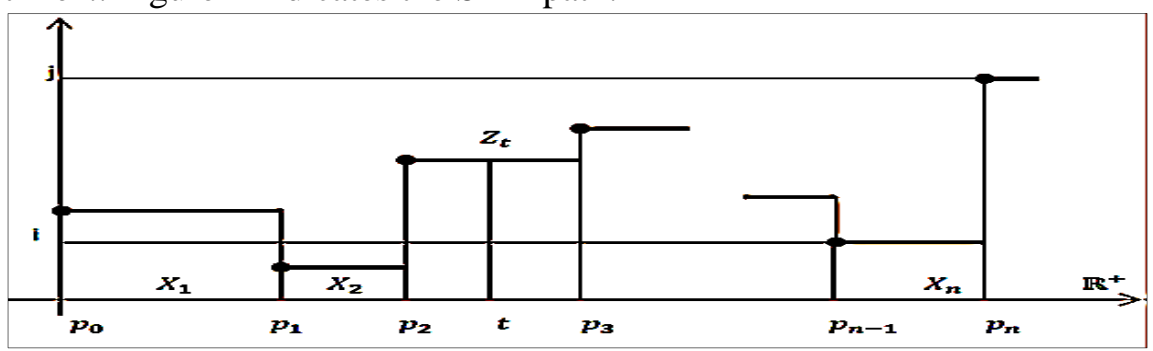

Figure 1. A schematic path of MRP and SMP

Suppose that in this model the initial state is $A M P_{0}$ in time $t_{0}$ (Log Amplitude abbreviated to "AMP"). Next, the system moves to other state and stay there for a length of time, etc. (Figure 2).

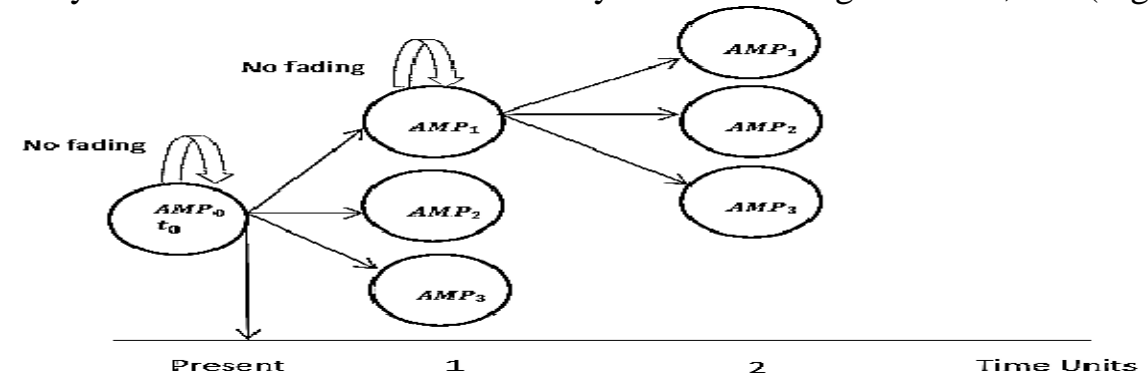

Figure 2. A Schematic model of a trajectory of the SMP for fading occurrences

By considering the last deep fading occurred with amplitude $A M P_{0}$ and the elapsed time since its happening, in Semi Markov Model, the next unit of time, the system may either have no deep fading or make a transition to any of other states $A M P_{1}, A M P_{2}$ or $A M P_{3}$. The SMP suggests that the likelihood of the next deep fading with special amplitude depends on the amplitude of the previous deep fading. The definitions of the basic elements of the SMP are as follows:

(i) The consecutive state occupancies of the SMP are managed by transition probabilities. The transition probability $\left(P_{i j}\right) \geq 0$ is a probability that the SMP which entered state $i$ on its last transition will enter state $j \neq i$ on its next transition. The transition probability matrix $(P)$ has a unity row-sum:

$\sum_{j=1}^{N} P_{i j}=1$

where $N$ is the total number of states. 
(ii) Once the process has entered state $i$ and given it will transit to state $j$, it remains for a random time $\tau_{i j}$ in state $i$. The holding times $\tau_{i j}$ are positive integer variables which are identified by a probability mass function $T_{i j}$ for a transiting from state $i$ to $j$ :

$P\left\{\tau_{i j}=m\right\}=T_{i j}(m), \quad m=1,2, \ldots, M \quad i, j=1,2, \ldots, N$.

(iii) Applying the transition probability matrix $P$ and holding time matrix $T(m)=T_{i j}(m)$, define the core matrix $C(m)$ as the probability of the event that a process entering state $i$ at time zero make a transition to state $j \neq i$ after a holding time $m$ :

$C(m)=P \odot T(m) \quad \sum_{j=1}^{N} T_{i j}(m)=1 \quad \forall i \neq j$.

Summing over the row elements of $C(m)$ for the $i$ th state yields the waiting time probability mass function $W_{i}(m)$ :

$W_{i}(m)=\sum C_{i j}(m)$

The complementary cumulative probability distribution of the process leaving state $i$ at a time greater than $n$ is calculated as:

$>W_{i}(n)=\sum_{m=n+1}^{\infty}$ Sum of elements in $i-$ th row of $C(m)$.

(iv) The interval transition probability $F_{i j}(n)$ indicates the SMP multistep realization. Suppose that the process entered state $i$ at time 0 is in state $j$ at time $n$. It could transit to some intermediary state $k$ at time $m(0<m \leq n)$ before reaching state $j$ or the process never left the state $i$ during the interval $[0, n] . F(n)=\left[F_{i j}(n)\right]$ is a function of the transition probabilities and the state holding time mass functions can obtain recursively as:

$$
F(n)=>W(n)+\sum_{m=0}^{n} G \otimes T(m) F(n-m) ; \quad n=0,1,2, \ldots
$$

where $>W(n)$ is a diagonal matrix with its $i$-th element equal to $>W_{i}(n)$; moreover, the $F(0)$ is defined as equal as the kronecker delta. If the $F=\lim _{n \rightarrow \infty} F(n)$ exists, the asymptotic behavior of interval transition probabilities over long time intervals can be identified by the limiting interval transition probability matrix $F$.

\section{APPLICATION OF THE MODEL}

\subsection{Simulation Materials and Results}

When a fading occurred for a received signal during transmission, both its envelope and phase fluctuate over time. See [26-28] for studying the fading occurrence completely. The statistical properties of the fading intervals of the Rayleigh process were given in [29]. In this section, the MATLAB code was used for generating the impulse response of the Jakes filter. The pseudo-random set of Gaussian input was generated and it was filtered; moreover, the Pdf and Power Spectral Density (PSD) were shaped by the inverse transform method with a required level of accuracy. The simulated fading data occurred in wireless systems in which motion is present, including Jakes filter, Gaussian noise with maximum Doppler $f_{d}=100$, sampling frequency $f_{s}=16 * f_{d}, t_{s}=1 / f_{s}$ and hence $\left(t_{s}\right)=6.2500 e-04 \mu \mathrm{sec}$. The time vector $\left[1 * t_{s}: t_{s}: 128 *\right.$ $\left.t_{s}\right]$ was used. The implementation of the Jakes filter was done with 128 points and the FFT block size 512. The simulation needs the generation of a process having the power spectral density

$S(f)=\left\{\begin{array}{lr}\frac{1}{\sqrt{1-\left(f^{f} / f_{d}\right)^{2}}}, & |f|<f_{d} \\ 0 & \text { o.w. }\end{array}\right.$.

The filter transfer function is 
$H(f)=\left\{\begin{array}{cl}{\left[1-\left(f / f_{d}\right)^{2}\right]^{-\frac{1}{4}}} & |f|<f_{d} . \\ 0 & \text { o.w. }\end{array}\right.$

The estimated PSD at the filter output and the fading envelope of this system presents in Figure 3.
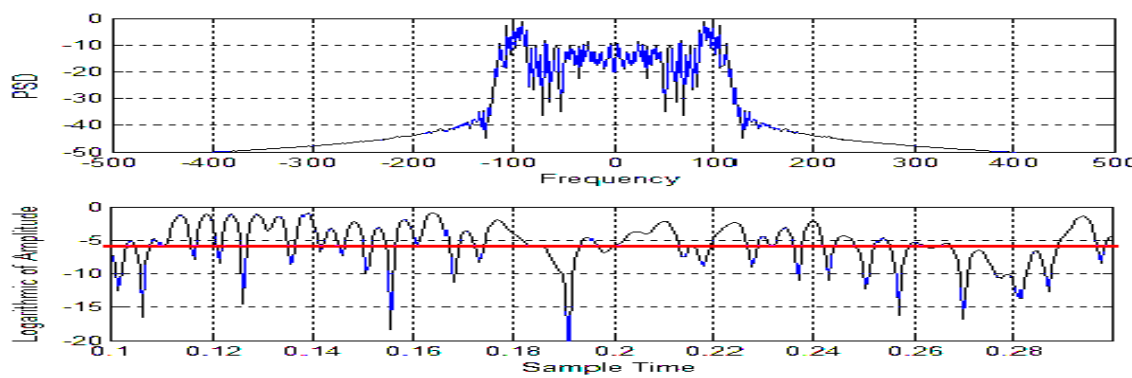

Figure 3. The estimated PSD at the filter output and the received signal strength envelope

The PSD is the frequency response of a random or periodic signal. It shows where the average power distributes as a function of frequency; moreover, the multipath fading is due to alternations in the atmosphere which are themselves random, therefore the forecasting of fading occurrence or distribution can only be expressed by statistical methods.

\subsection{The Estimated Semi Markov Model}

A common statistical model for the signal strength of communication channels is the stochastic process. A probabilistic stochastic model which is advantageous in complex dynamic systems is the SMP. It can be seen from the Figure 3 that the fading occurrence is highly variable and the SMP is more suitable for analyzing the signal behavior since it can represent with more precision the reality of a fading in which the envelope continuously changes over time. Fading occurrences in time intervals are not independent of each other. Therefore, the SMP can be employed which considers the dependence of all fades in time. Fades of a signal are dependent on each other and the times of successive fades are related to each other. The SMP forecasts time and amplitude, simultaneously. According to the signal amplitude observations during a time interval, the SMP partitioned the received data into three states. This model has the basic Markov property of one step memory and besides the calculation of the transition probabilities; it provides the duration of times spent in any state. The holding time between successive fades depends on the amplitude of the previous and the next fades. Hence, this model gives knowledge of the duration of fades. Calculating the interval transition probabilities indicates the future behavior of the signal and the fade occurrence time. The time and amplitude state of the next fading can be obtained by the SMP. This prediction is beneficial for reducing the detrimental impact of fading on transmission performance. The SMP is a flexible stochastic model which enables more accurate prediction and simulation of system performance and availability. The 142 deep fades which are lower than the mean log amplitudes were considered for modeling. In this section, the nonparametric estimation of the Semi Markov model was applied. A stochastic process is considered to be a collection of random variables. Each random variable in the collection takes value from the same mathematical space which is called the state space. The state space can be partitioned according to the different rules which in our analysis the "equidistant partitioning" method was regarded. All the amplitude values which constitute the sample space were partitioned in three states as equation (13). Then we identified that each amplitude value belongs to which of these states. The probabilities of moving from state $i=1,2,3$ to $j=1,2,3$ are obtained by calculating the transition probability matrix. It is calculated by the ratio of the number of $i$ to $j$ transitions to the total number of transitions from $i$. We have applied the Semi Markov Model and regarded state boundaries according to the equidistant partitioning technique, the minimum and maximum of the log amplitudes as follows:

$S_{1}:-22.1258 \leq A M P_{S}<-16.5193$

$S_{2}:-16.5193 \leq A M P_{S}<-10.9128$

$S_{3}:-10.9128 \leq A M P_{S}<-5.3063$.

The transition probability matrix of amplitude to amplitude was obtained as: 
$P=\left[\begin{array}{ccc}0 & 0.25 & 0.75 \\ 0.125 & 0.25 & 0.625 \\ 0.017 & 0.092 & 0.891\end{array}\right]$

This matrix is satisfied in equation (5). In other words the row sums of this matrix are one. It is concluded that all states desired to visit the state $S_{3}$ and deep fading with $S_{3}:-10.9128 \leq A M P_{S}<-5.3063$ is most probable. Moreover, the state $S_{1}$ does not tend to to remain in the same state $S_{1}$. The holding time mass functions are computed by regarding the time elapsed between succeeding fading occurrences according to equation (6) (Table 1). One unit time is taken into account as $t_{s}=0.0006 \mu s e c$. The highest time interval for the log amplitude transitions was found to be 21 unit times.

Table 1. Holding time mass functions of the amplitude to amplitude transitions

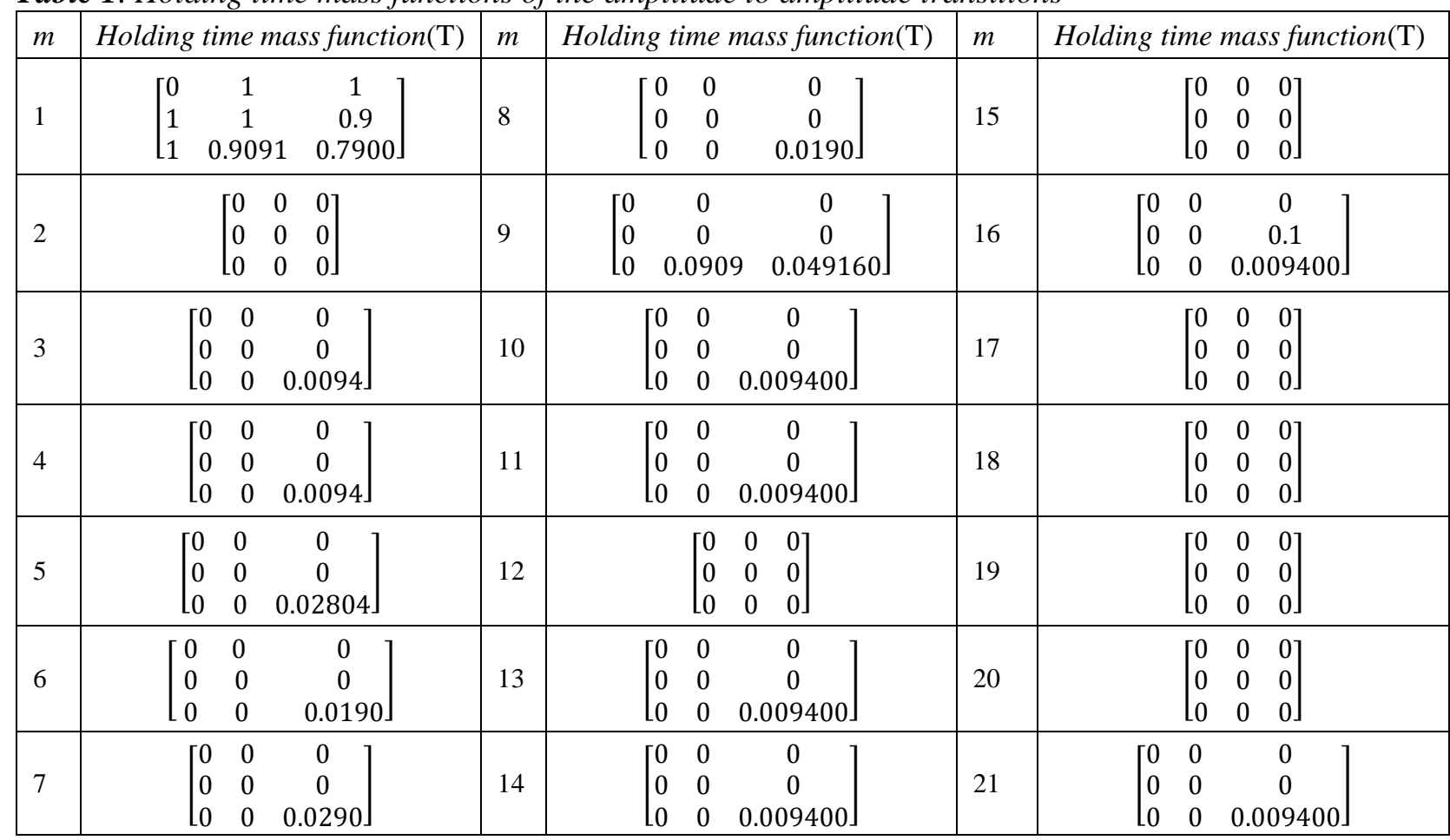

It can be seen from Table 1 that before the transition from state $i=1,2,3$ to $j=1,2,3$, the amplitude remains in the state $i$ for a time $t_{i j}=1, \ldots, 21$ unit time. For calculating $T(1)$, the ratio of the number of $i$ to $j$ transitions in one unit time to the all transition from $i$ to $j$ is calculated. For calculating $T(2)$, the ratio of the number of $i$ to $\mathrm{j}$ transitions in two unit times $\left(t_{s}=0.0012 \mu \mathrm{sec}\right)$ to the all transition from $i$ to $j$ is calculated, etc. It can conclude that with 0.9 probability the process stays one unit time $\left(t_{s}=0.0006\right)$ in state $S_{2}$ before going to the state $S_{3}$; moreover, with 0.090 probability the process stays 9 unit time in the state $S_{3}\left(-10.9128 \leq A M P_{S}<-5.3063\right)$ before going to the state $S_{2}\left(16.5193 \leq A M P_{S}<-10.9128\right)$. We must specify both the holding time mass functions and the transition probabilities to describe a discretetime semi-Markov process completely. These values are beneficial in calculating the interval transition probabilities.

The cumulative probabilities of waiting time distributions of the log amplitude are shown in Figure 4 for the three states. The probability of the amplitude remaining $\leq n$ time units in each state can be shown by the length of stay in that state. 


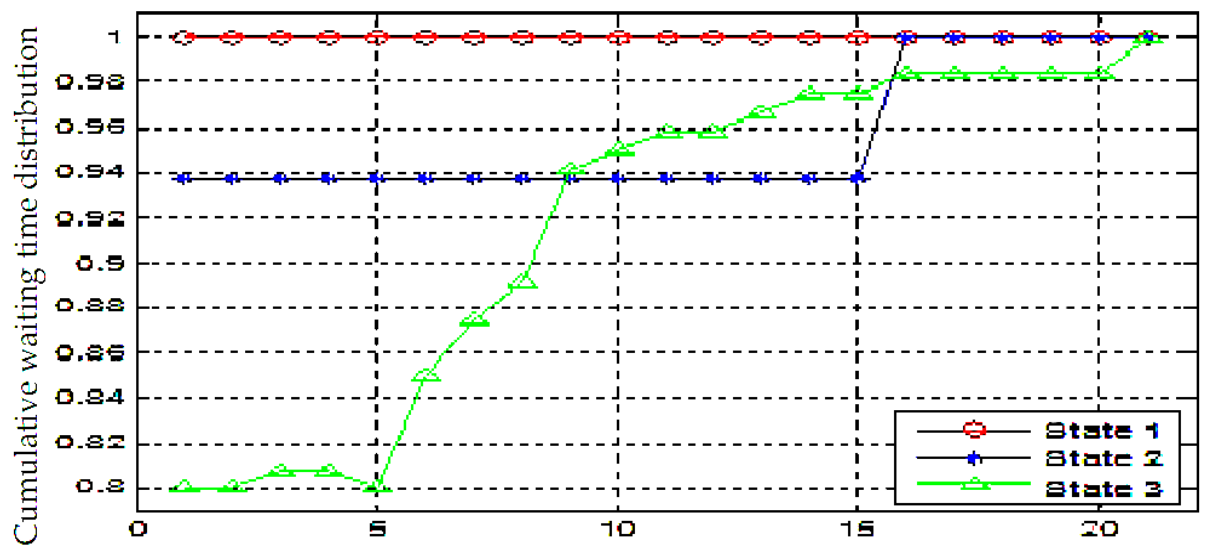

Unit times

Figure 4. Cumulative waiting time distributions of the three states

It is concluded from Figure 4 that the cumulative probability of staying the amplitude in state $S_{2}$ for the $\leq 5$ time units is approximately 0.8. All the staying probabilities for $\leq n$ time units for $S_{1}, S_{2}$ and $S_{3}$ can be seen from this figure; moreover, it is evident that all these trends reach to probability one after 21 unit times. The interval transition probabilities by equation (10) were calculated for the next 21 unit times (Table 2) by calculating the core matrix and the complementary cumulative probability distribution by equation (7) and (9), respectively. For calculation $\mathrm{F}(1)$, according to equation (10) we compute $>W(1)+$ $\sum_{m=0}^{n} G \otimes T(0) F(0)$, where $\mathrm{T}(0)$ is zero. For calculating $\mathrm{F}(2)$, we compute $>W(2)+$ $G \otimes T(0) F(1)+G \otimes T(1) F(0)$, etc.

Table 2. Interval transition probabilities of the amplitude to amplitude transitions

\begin{tabular}{|c|c|c|c|c|c|c|c|c|c|c|c|}
\hline$m$ & \multicolumn{3}{|c|}{ Interval transition probabilities $(F)$} & \multirow{2}{*}{$\begin{array}{l}m \\
8\end{array}$} & \multicolumn{3}{|c|}{ Interval transition probabilities $(F)$} & \multirow{2}{*}{$\begin{array}{l}m \\
15\end{array}$} & \multicolumn{3}{|c|}{ Interval transition probabilities $(F)$} \\
\hline 1 & {$\left[\begin{array}{c}0 \\
0.125 \\
0.01666\end{array}\right.$} & $\begin{array}{c}0.25 \\
0.3125 \\
0.0834\end{array}$ & $\left.\begin{array}{c}0.75 \\
0.5625 \\
0.9\end{array}\right]$ & & {$\left[\begin{array}{c}0.0115 \\
0.011 \\
0.010\end{array}\right.$} & $\begin{array}{l}0.088 \\
0.149 \\
0.067\end{array}$ & $\left.\begin{array}{c}0.9005 \\
0.840 \\
0.923\end{array}\right]$ & & {$\left[\begin{array}{l}0.0112 \\
0.0105 \\
0.0114\end{array}\right.$} & $\begin{array}{l}0.1097 \\
0.1686 \\
0.0935\end{array}$ & $\left.\begin{array}{l}0.8791 \\
0.8206 \\
0.8951\end{array}\right]$ \\
\hline 2 & {$\left[\begin{array}{l}0.0438 \\
0.0407 \\
0.0221\end{array}\right.$} & $\begin{array}{c}0.1406 \\
0.21870 \\
0.0885\end{array}$ & $\left.\begin{array}{l}0.8156 \\
0.7406 \\
0.8894\end{array}\right]$ & 9 & {$\left[\begin{array}{l}0.0107 \\
0.0102 \\
0.0103\end{array}\right.$} & $\begin{array}{c}0.0881 \\
0.149 \\
0.077\end{array}$ & $\left.\begin{array}{c}0.9122 \\
0.850 \\
0.930\end{array}\right]$ & 16 & {$\left[\begin{array}{l}0.0111 \\
0.0104 \\
0.0113\end{array}\right.$} & $\begin{array}{l}0.1123 \\
0.1084 \\
0.0960\end{array}$ & $\left.\begin{array}{l}0.8766 \\
0.8812 \\
0.9191\end{array}\right]$ \\
\hline 3 & {$\left[\begin{array}{l}0.0267 \\
0.0280 \\
0.0193\end{array}\right.$} & $\begin{array}{l}0.1211 \\
0.1846 \\
0.0822\end{array}$ & $\left.\begin{array}{c}0.8522 \\
0.7874 \\
0.902\end{array}\right]$ & 10 & {$\left[\begin{array}{l}0.0103 \\
0.0973 \\
0.0117\end{array}\right.$} & $\begin{array}{l}0.0955 \\
0.1545 \\
0.0820\end{array}$ & $\left.\begin{array}{c}0.9103 \\
0.850 \\
0.930\end{array}\right]$ & 17 & {$\left[\begin{array}{l}0.0111 \\
0.0114 \\
0.0107\end{array}\right.$} & $\begin{array}{l}0.0991 \\
0.1003 \\
0.0897\end{array}$ & $\left.\begin{array}{l}0.8898 \\
0.8883 \\
0.8996\end{array}\right]$ \\
\hline 4 & {$\left[\begin{array}{l}0.018 \\
0.020 \\
0.015\end{array}\right.$} & $\begin{array}{c}0.1080 \\
0.170 \\
0.075\end{array}$ & $\left.\begin{array}{l}0.874 \\
0.810 \\
0.920\end{array}\right]$ & 11 & {$\left[\begin{array}{l}0.0112 \\
0.0103 \\
0.0120\end{array}\right.$} & $\begin{array}{c}0.100 \\
0.1592 \\
0.0853\end{array}$ & $\left.\begin{array}{l}0.9198 \\
0.8305 \\
0.9027\end{array}\right]$ & 18 & {$\left[\begin{array}{l}0.0109 \\
0.0116 \\
0.0110\end{array}\right.$} & $\begin{array}{l}0.0924 \\
0.0935 \\
0.0891\end{array}$ & $\left.\begin{array}{l}0.8967 \\
0.8949 \\
0.8999\end{array}\right]$ \\
\hline 5 & {$\left[\begin{array}{l}0.011 \\
0.017 \\
0.014\end{array}\right.$} & $\begin{array}{c}0.099 \\
0.16 \\
0.070\end{array}$ & $\left.\begin{array}{c}0.89 \\
0.83 \\
0.916\end{array}\right]$ & 12 & {$\left[\begin{array}{l}0.0116 \\
0.0107 \\
0.0121\end{array}\right.$} & $\begin{array}{l}0.1038 \\
0.1628 \\
0.0881\end{array}$ & $\left.\begin{array}{l}0.8846 \\
0.8265 \\
0.8998\end{array}\right]$ & 19 & {$\left[\begin{array}{l}0.0111 \\
0.0117 \\
0.0111\end{array}\right.$} & $\begin{array}{c}0.09020 \\
0.09020 \\
0.0884\end{array}$ & $\left.\begin{array}{c}0.8987 \\
0.852 \\
0.9005\end{array}\right]$ \\
\hline 6 & {$\left[\begin{array}{c}0.0140 \\
0.014 \\
0.0123\end{array}\right.$} & $\begin{array}{c}0.0930 \\
0.154 \\
0.0679\end{array}$ & $\left.\begin{array}{c}0.893 \\
0.832 \\
0.9198\end{array}\right]$ & 13 & {$\left[\begin{array}{l}0.0117 \\
0.0109 \\
0.0114\end{array}\right.$} & $\begin{array}{l}0.1068 \\
0.1657 \\
0.0878\end{array}$ & $\left.\begin{array}{l}0.8815 \\
0.8234 \\
0.9008\end{array}\right]$ & 20 & {$\left[\begin{array}{l}0.0112 \\
0.0105 \\
0.0110\end{array}\right.$} & $\begin{array}{l}0.0888 \\
0.0835 \\
0.0873\end{array}$ & $\left.\begin{array}{c}0.9 \\
0.906 \\
0.9017\end{array}\right]$ \\
\hline 7 & {$\left[\begin{array}{l}0.0129 \\
0.0124 \\
0.0112\end{array}\right.$} & $\begin{array}{c}0.0897 \\
0.151 \\
0.0669\end{array}$ & $\left.\begin{array}{l}0.8974 \\
0.9725 \\
0.9219\end{array}\right]$ & 14 & {$\left[\begin{array}{l}0.0113 \\
0.0106 \\
0.0114\end{array}\right.$} & $\begin{array}{l}0.1073 \\
0.1667 \\
0.0906\end{array}$ & $\left.\begin{array}{c}0.8814 \\
0.8227 \\
0.898\end{array}\right]$ & 21 & {$\left[\begin{array}{l}0.0109 \\
0.0111 \\
0.0110\end{array}\right.$} & $\begin{array}{l}0.0863 \\
0.0855 \\
0.0868\end{array}$ & $\left.\begin{array}{l}0.9028 \\
0.9034 \\
0.9022\end{array}\right]$ \\
\hline
\end{tabular}


It can be deduced from the Table 2 that after one to 21 unit times, the amplitude of the signal tends to go to which state $\left(S_{1}, S_{2}\right.$, and $\left.S_{3}\right)$. These probabilities are beneficial in preventing fading occurrences. In a long time after 21 unit times, it can be seen that if a deep fading occurred in $S_{1}$, the next deep fading will happen in $S_{1}, S_{2}, S_{3}$ with the probability $0.0109,0.0863$ and 0.909028 , respectively. It concludes that all states in long times will prefer to visit the state with $A M P_{3}$. We forecasted all probabilities of deep fading occurrences with $A M P_{3}<-5.3063$ from 1 to 21 next unit times, which equals the inverse of the sampling frequency. If a deep fading occurred in $A M P_{3}$, the next deep fading in one unit time will happen in $A M P_{1}, A M P_{2}, A M P_{3}$ with probability $0.02,0.08$ and 0.9 , respectively. Supposing that the last occurrence is in the $A M P_{1}$, the probabilities of next deep fading occurrence after 21 unit times with $A M P_{1}, A M P_{2}, A M P_{3}$ are $0.01,0.09$ and 0.90 , respectively. These interval transition probabilities were plotted versus time unit in Figure 5 .
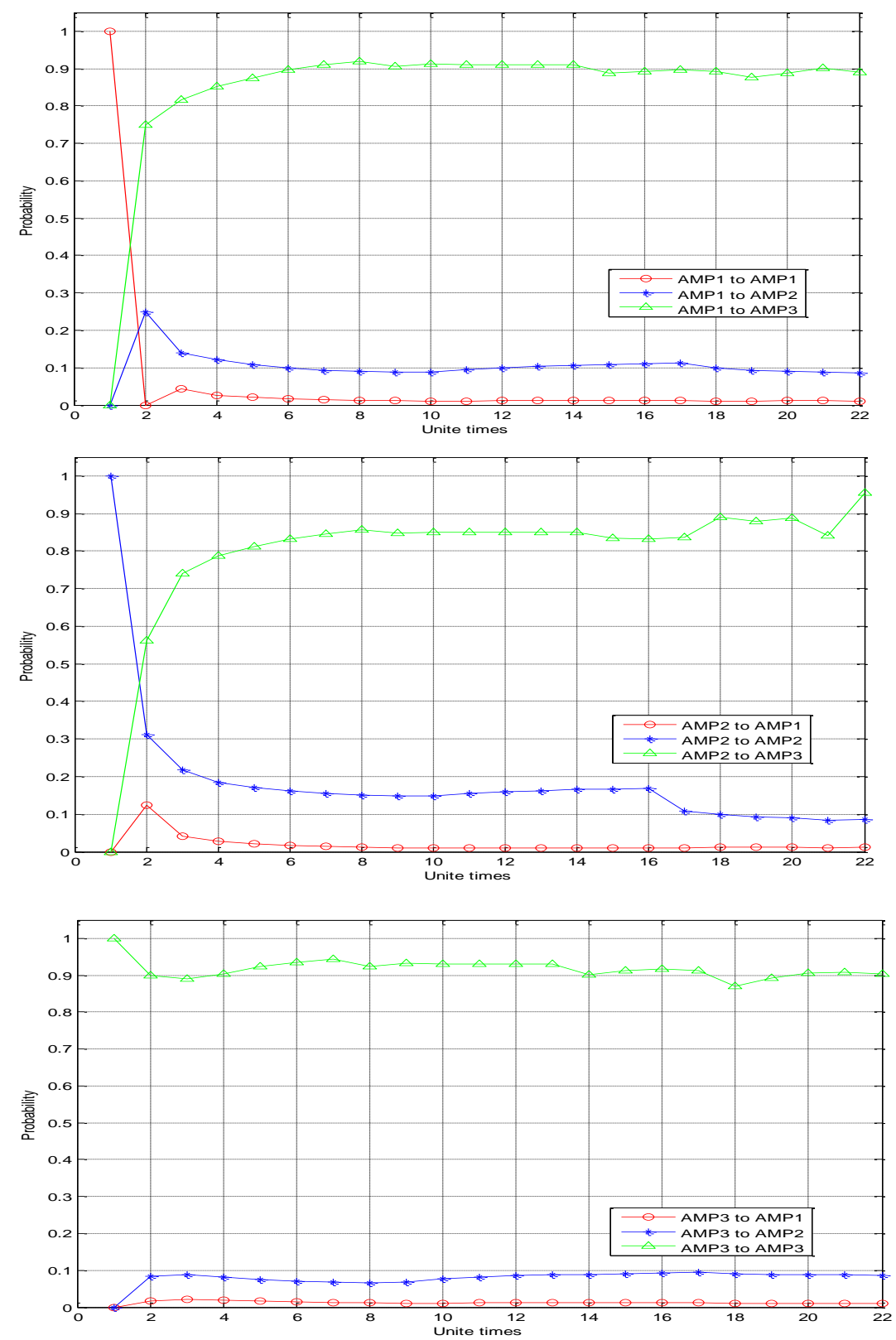

Figure 5. $F(n)$ 's for the amplitude to amplitude transitions

From Figure 5, it is deduced that the probabilities of transition from all states to $A M P_{3}$ are the highest; moreover, the trends of $A M P_{1}$ and $A M P_{2}$ to $A M P_{2}$ are increasing with time. However, the trends of $A M P_{1}$ and $A M P_{2}$ to $A M P_{2}$ is decreasing with time. The $A M P_{1}$ to the $A M P_{1}$ trend is to some extent uniform and it 
fluctuated slightly. These probabilities are helpful in improving the reliable communication channels in transmission by forecasting the deep fading occurrences amplitude along with their time. We can see from this figure that which amplitude state is the most probable for the fading occurrence in a future time. If the signal amplitude is in state $S_{1}$ after two unit times, it is most probable to go to the state $S_{3}$; moreover, the probability of remaining in the same state $S_{1}$ is the least. If the signal amplitude is in state $S_{2}$ or state $S_{3}$ then after $m$ unit time going to which state was forecasted.

This paper is newly discovered in modeling wireless fading occurrences. In comparison to the Markov model it is concluded that the time spent in any state in the Markov model has to follow only an exponential distribution, and that may result in unrealistic values of the duration of the states but in our Semi Markov model the holding time which shows the time spent in any state has an arbitrary holding time distribution which appropriates to the data. This model gives realistic forecasted values. It can claim that the SMP is a generalization of Markov process. This model estimates the interval transition probabilities which indicate the transitions between states of the system with respect to time.

\section{CONCLUSIONS}

Wireless communication is one of the most significant fields in the communication area. One of the most troublesome issues in wireless communication is fading. Predicting the fading occurrences can improve the reliable transmitter/receiver and the power control in wireless systems. At this time, it is attempted to forecast the behavior of communication systems over a fading channel. There is a wide range of precise and simple statistical models for modeling fading channels. In this paper, we proposed the Semi-Markov Model for predicting the deep fading occurrence probabilities with Jakes filter. The high accuracy and low computation time make the SMP suitable for forecasting the fading occurrence amplitude and time. The transition probability matrix, the holding time mass functions and the interval transition probability matrices are the main equations which indicate the probabilities of transitions between states, the probability of remaining in a specified state before moving to another state and the probability of transition after some unit times, respectively. In this paper, the simulated fading data have been used which occurred in wireless systems in which motion is present including Jakes filter and Gaussian noise. The implementation of the Jakes filter was done with 128 points and the FFT block size 512. By employing occurred deep fades with amplitude lower than a mean of the amplitudes, the transition probability matrix and the holding time mass functions were calculated for the next 1 to 21 unit times; moreover, the interval transition probabilities for the amplitude to amplitude transition were obtained according to the core matrix and the cumulative waiting time probability mass functions. The probabilities of fading occurrence in $A M P_{3}$ following the last deep fading in $A M P_{1}$ and $A M P_{2}$ increase with time. The probabilities of fading occurrence in $A M P_{2}$ following $A M P_{1}$ and $A M P_{2}$ decrease with time. The $A M P_{1}$ to the $A M P_{1}$ trend is to some extent uniform and it fluctuates slightly. The probabilities of transition from all states to $S_{3}\left(:-10.9128 \leq A M P_{S}<-5.3063\right)$ are the highest. We can conclude which amplitude state is most probable for the fading occurrence in a long time. The prediction with respect to the time is essential in wireless networks which is possible by applying the Semi Markov model.

\section{CONFLICTS OF INTEREST}

No conflict of interest was declared by the authors.

\section{REFERENCES}

[1] Zheng, Z., Trivedi, K. S., Qiu, K., Xia, R., "Semi Markov models of composite web services for their performance, reliability and bottlenecks," IEEE Transactions on Services Computing, 10(3): 448-460, (2017).

[2] Grabski, F., Semi Markov processes: Applications in system reliability and maintenance, Elsevier Inc., (2015). 
[3] D’Amico, G., Petroni ,F., "A Semi Markov model for price returns," Physica A: Statistical Mechanics and its Applications,391(20): 4867-4876, (2012).

[4] Wanneveich,M., Jacqmin-Gadda, H., Dartigues, J., Joly P., "Prediction of health indicators for chronic disease under a semi Markov assumption," Theoretical Population Biology, 119: 83-90, (2018).

[5] Ghosh, S., Gosavi, A., "A Semi-Markov model for post-earthquake emergency response in a smart city," Control Theory and Technology, 15(1): 13-25, (2017).

[6] Niemeyer, A., "Safety margins systematic biometric and financial risk in a semi Markov life insurance framework," Risks, 3: 36-60, (2015).

[7] Sengathir, A., Manoharan, R., "A futuristic trust coefficient-based semi-Markov prediction model for mitigating selfish nodes in MANETs," EURASIP Journal on Wireless Communications and Networking, 1, 1-13, (2015).

[8] Vishnevskii, V. M., Andronov, A. M., "Estimating the throughput of wireless hybrid systems operating in a semi-Markov stochastic environment," Automation and Remote Control,78(12): 21542164, (2017).

[9] Yin, R., Liu, B., Liu, H., Li, Y., Dong M., "A quantitative fault tolerance evaluation model for topology in wireless sensor networks based on the semi Markov process," Neurocomputing, 149: 1014-1020, (2015).

[10] Chen, M., Ekman, T., Viberg, M., "New approaches for channel prediction based on sinusoidal modeling," EURASIP Journal on Advances in Signal Process, 2007(1):2-13, (2006).

[11] Eyceoz, T., Duel-Hallen, A., Hallen, H., "Deterministic Channel Modeling and Long Range Prediction of Fast Mobile Radio Channels," IEEE Communication Metters, 2(9): 254-256, (1998).

[12] Schiavone, J. A., Hermiller, S. M., "A Regression model for forecasting Microwave Radio fading at Palmetto, GA," IEEE Transactions on Antennas and Propagation,AP-34(7): 936-942, (1986).

[13] Lavanya, V., Sasibhushana, Rao G., Bidikar B., "Fast fading mobile channel modeling for wireless communication," Procedia Computer Source, 85: 777-781, (2016).

[14] Skima, M. A., Ghariani, H., Lahiani, M., "A multi criteria comparative analysis of different Rayleigh fading channel simulators," AEU Int. J. Electron. Commun, 66(6): 550-560, (2014).

[15] Blaunstein, N., Cohen, V, Hayakawa, M., "Prediction of fading phenomena in land-satellite communication links," Radio Science, 45(6): 1-13, (2010).

[16] Rodhiah, H. A., Hamid, E. Y., "ARMA model for multipath Rayleigh fading using Minimum Description Length criterion," in 10th International Conference on Telecommunication Systems Services and Applications(TSSA), (2016).

[17] Olofsson, T., Ahlen, A., Gidlund, M., "Modeling of the fade statistics of wireless sensor network channels in industrial environments," IEEE Transactions on Signal Processing, 64(12): 3021-3034, (2016). 
[18] Lutz, E., Cygan, D., Dippold, M., Dolainsky, F., Papke, W., "The land mobile satellite communication channel-recording, Statistics and channel model," IEEE Trans. Veh. Techol., 40: 375-386, (1991).

[19] Lin, H. P., Tsai, F. S., Tseng, M. J., "Satellite propagation channel modeling using photogrammetry and hidden Markov model approach," IEEE Proc. Microw. Antennas Propag., 148(6): 550-560, (2001).

[20] Sajadieh, M., Kschischang, F. R., Len-Garciae ,A., "A block memory model for correlated Rayleigh fading channels," in Proceedings of ICC/SUPERCOMM 96-International Conference on Communications, (1996).

[21] Iglesias, D.R., Sanchez, M.G., "Semi Markov Model for Low-Elevation Satellite-Earth Radio Propagation Channel," IEEE Transactions on Antennas and Propagation, 60(5): 2481-2490, (2012).

[22] Wang, J., Cai J., Alfa A. S., "New Channel Model for Wireless Communications: Finite-State PhaseType Semi-Markov Channel Model," in IEEE International Conference on Communications, May (2008).

[23] Georgiadis, S., Limnios ,N., "Nonparametric estimation of the stationary distribution of a discrete time semi Markov process," Communication in Statistics-Theory and Methods, 44(7): 1319-1337, (2015).

[24] Girardin, V., Limnios, N., Applied Probability from Random Sequences to Stochastic Processes, Springer Nature Switzerland AG, (2018).

[25] Devolder, P., Janssen, J., Manca, R., "Homogeneous and non-homogeneous semi Markov models," Basic Stochastic Processes, 113-163, (2015).

[26] Ekwe, O. A., Abioye, V, Oluwe, M.O., Okoro, K.C., "Effective Fading Reduction Techniques in Wireless Communication System," OSR Journal of Electronics and Communication Engineering, 9(4): 35-43, (2014).

[27] Sachdeva, N., Sharma D., "Diversity: A Fading Reduction Technique," International Journal of Advanced Research in Computer Science and Software Engineering, 2(6), (2012).

[28] Özbek, B., Ruyet, D. L., Feedback Strategies for Wireless Communication, Springer-Verlag New York, (2014).

[29] Zhang, H., Liu, Y., Gao, J., "Statistical Analysis of Wireless Fading Channels," in International Conference on Artificial Intelligence and Computational Intelligence: Artificial Intelligence and Computational Intelligence, 6320: 385-366, (2010). 